\title{
Deletion of ACTA2 in mice promotes angiotensin II induced pathogenesis of thoracic aortic aneurysms and dissections
}

\author{
Jiancheng Cheng ${ }^{1}$, Xianwu Zhou ${ }^{2}$, Xionggang Jiang ${ }^{3 \#}$, Tucheng Sun ${ }^{4 \#}$ \\ ${ }^{1}$ Department of Cardiothoracic Surgery, Zhengzhou Central Hospital Affiliated to Zhengzhou University, Zhengzhou 450007, China; ${ }^{2}$ Department \\ of Cardiovascular Surgery, Wuhan Asia Heart Hospital, Wuhan 430015, China; ${ }^{3}$ Department of Cardiovascular Surgery, Union Hospital, Tongji \\ Medical College, Huazhong University of Science and Technology, Wuhan 430022, China; ${ }^{4}$ Guangdong Cardiovascular Institute, Guangdong \\ General Hospital, Guangdong Academy of Medical Sciences, Guangzhou 510080, China \\ Contributions: (I) Conception and design: J Cheng; (II) Administrative support: T Sun, X Jiang; (III) Provision of study materials: X Zhou; (IV) \\ Collection and assembly of data: J Cheng; (V) Data analysis and interpretation: J Cheng; (VI) Manuscript writing: All authors; (VII) Final approval of \\ manuscript: All authors. \\ \#These authors contributed equally to this work. \\ Correspondence to: Xionggang Jiang. Department of Cardiovascular Surgery, Union Hospital, Tongji Medical College, Huazhong University of Science \\ and Technology, Wuhan 430022, China. Email: jiangxionggang@hotmail.com; Tucheng Sun. Guangdong Cardiovascular Institute, Guangdong \\ General Hospital, Guangdong Academy of Medical Sciences, Guangzhou 510080, China. Email: suntucheng@126.com.
}

Background: Mutation of the ACTA2 ( $\alpha-2$ smooth muscle actin) gene accounts for $\sim 15 \%$ of all cases of familial thoracic aortic aneurysms and dissections. Surprisingly, no severe vascular phenotypes were observed at baseline in mice carrying this gene mutation. Our aim was to explore whether mutation of ACTA2 promotes the development of aneurysms or dissections in the presence of angiotensin II (AngII) and to determine whether this mutation has an impact on the phenotypic modulation and apoptosis mediated by AngII in vascular smooth muscle cells (VSMCs).

Methods: Mice were divided into three groups: AngII stimulated-wild-type (WT) (AngII) and ACTA2 ${ }^{-/}$ mice (ACTA2) group, in which AngII were administered subcutaneously into 8-week-old C57 mice and $\mathrm{ACTA}^{-/-}$mice, respectively, for 4 weeks using osmotic minipumps, and the control group (WT), in which the WT mice were infused with normal saline (NS). Ultrasound was performed to quantify lumen diameters. RT-qPCR and Western blot were used to assess gene expression, and histobiochemistry was used to evaluate the pathological changes in the thoracoabdominal aortas. TUNEL was used to assess apoptosis in VSMCs.

Results: Compared with the AngII- group, the ACTA2 mice exhibited more severity of dilated lumena of the aortas, a significantly increased expression of osteopontin (OPN), an elevated ratio of Bax/Bcl-2, increased apoptosis, and a decreased expression of $\alpha$-smooth muscle actin ( $\alpha$-SMA).

Conclusions: Knockout of ACTA2 promoted AngII induced progressive lumen dilation of the aortas, apoptosis, and the phenotypic modulation in VSMCs in mice.

Keywords: Aortic aneurysm; aortic dissection; smooth muscle actin alpha 2 (ACTA2); gene mutation

Submitted Oct 20, 2017. Accepted for publication Jul 09, 2018.

doi: $10.21037 /$ jtd.2018.07.75

View this article at: http://dx.doi.org/10.21037/jtd.2018.07.75

\section{Introduction}

Aortic aneurysms and dissections have a high morbidity and mortality worldwide $(1,2)$. The most prevalent genetic cause of familial thoracic aortic aneurysms and dissections is the mutation in vascular smooth muscle $\alpha$-actin encoded by the gene ACTA2 ( $\alpha-2$ smooth muscle actin). To date, more than 40 mutations in ACTA2 have been found and these mutations account for $\sim 15 \%$ of aneurysms and dissections diagnosed in clinics (3-5). However, mouse models that either involve the deletion of ACTA2 $\left(\mathrm{ACTA2}^{-/}\right)$or the use 
Table 1 Primary antibody and secondary antibody

\begin{tabular}{ll}
\hline Antibody & Company \\
\hline Bax rabbit mAb & America Cell Signaling Company \\
Bcl-2 (D17C4) rabbit mAb & America Cell Signaling Company \\
Rabbit anti- $\alpha$-SMA antibody & America Genetex Company \\
Rabbit anti-OPN antibody & America Abcam Company \\
Rabbit anti mouse IgG/HRP & Tianjin Sanjian Company \\
$\begin{array}{l}\text { Rabbit/mouse anti-CD68 } \\
\text { antibody }\end{array}$ & Wuhan Doctoral Company \\
\hline
\end{tabular}

$\alpha$-SMA, $\alpha$-smooth muscle actin; OPN, osteopontin; HRP, horseradish peroxidase.

of a point mutation on the Myh11 gene (Myh11 $1^{\mathrm{R} 247 \mathrm{C} / \mathrm{R} 247 \mathrm{C}}$ ) do not develop any discernable vascular phenotype at baseline (6).

We hypothesized that ACTA2 is involved in the pathogenesis of aortic aneurysms and dissections in mice, in response to stressors. In the present study, we used an ACTA2 loss of function mouse model and investigated the role of ACTA2 in the development of aortic aneurysms and dissections in the presence of angiotensin II (AngII), a chemical that is widely used to induce aortic aneurysms and dissections in mice (Curr Pharm Des 2015;21:4049-60).

\section{Methods}

\section{Animal models}

All animal procedures were approved by the Committee Tongji Medical College, Huazhong University of Science and Technology (ethical card: IACUC No.: 630). A mouse germline ACTA2 mutation was generated in a mixed C57BL/6J background in Cyagen Biosciences of Guangdong. All mice were maintained in a pathogen-free environment and had free access to water and normal laboratory diet. Male wild-type (WT) and homozygous ACTA2 mutant $\left(\mathrm{ACTA}^{--}\right)$mice were divided into three groups at 8 weeks of age (10 mice per group): AngII- stimulated WT (ANgII) and AngII stimulated ACTA2 ${ }^{--}$(ACTA2) groups, in which mice were infused with AngII (1,000 ng/ $/ \mathrm{kg} / \mathrm{min})$, respectively, for 4 weeks using osmotic minipumps, and the control group (WT), in which normal saline (NS) was infused into WT mice (NS group). Infusion was performed using Alzet osmotic pumps (model 2004, Durect Corp; Cupertino, CA, USA), which were implanted subcutaneously on the right flank through an incision of the scapular region.

\section{Ultrasound imaging}

On day 14 of AngII or NS infusion, abdominal aortas of all mice were scanned by high-frequency ultrasound (Vevo 2100, Visual Sonics, Toronto, Canada), and aortic lumen diameters were measured as an index of vascular expansion.

\section{Tissue extract}

The thoracoabdominal aorta from the mice that died suddenly during the course of the experiment were collected and analyzed for gene expression and histology. In all other mice, infusion of AngII or NS was performed for 28 days. Following this, the mice were sacrificed and the thoracoabdominal aortas were collected for gene expression and histological analysis.

\section{Western blot}

Twenty-five micrograms of total protein purified was resolved from the thoracoabdominal aorta using SDSPAGE. The purified protein was then transferred onto a polyvinylidene fluoride (PVDF) membrane. The membrane was probed with the primary antibodies of interest and secondary anti-rabbit-HRP (Table 1). Specific protein bands were detected using ECL (Beyotime Biotechnology).

\section{Real-time polymerase chain reaction (PCR)}

The tissues were homogenized in Trizol (Invitrogen) and subjected to total RNA purification. Reverse transcription (RT) reactions were performed using the RT Master Mix system (ABM Inc., New York, USA). Real-time PCR was carried out using SYBR Green PCR mix (ABM Inc., New York, USA). Reactions were run in triplicate for three independent experiments. The expression level of the housekeeping gene GAPDH was used as an internal control. Expression data was normalized to that of GAPDH and analyzed by the quantitation-comparative $\mathrm{C}_{\mathrm{T}}$ settings. The sequences of the primers that were used in this study are listed in Table 2.

\section{Histology}

Arterial segments were fixed in $10 \%$ paraformaldehyde and stored in $70 \%$ ethanol until all specimens were embedded in paraffin and sectioned. Standard hematoxylin \& eosin (H\&E), Verhoeff-Van Gieson (VVG), and Masson's stains 
Table 2 Primer sequences of PCR

\begin{tabular}{llll}
\hline Molecule & Primer & Base sequence & Base number \\
\hline ACTA2 & M-Acta2-F & CGAAACCACCTATAACAGCATCA & 181 \\
& M-Acta2-R & GCGTTCTGGAGGGGCAAT & 208 \\
Actin & M-actin-F & CTGAGAGGGAAATCGTGCGT & \\
& M-actin-R & CCACAGGATTCCATACCCAAGA & \\
\hline
\end{tabular}

PCR, polymerase chain reaction; ACTA2, smooth muscle actin alpha 2.

were used to assess the changes in cell morphology and the organization of extracellular constituents such as the elastic laminae, collagen fibers, and vascular smooth muscle cells (VSMC). Tunnel stain was used to evaluate the apoptosis of the aortic VSMCs. A microscope (BA310, Motic) equipped with an Olympus DP70 digital camera was used to acquire images of the stained aortic cross sections using a consistent setting.

\section{Statistical analysis}

Data are expressed as mean \pm standard error of the means (SEM). Student's $t$ tests or nonparametric tests were used to analyze the significance of the differences in the data between the groups. Statistical analyses were performed using GraphPad Prism, and $\mathrm{P}<0.05$ was considered to be statistically significant.

\section{Results}

Increased AngII induced progressive lumen dilation in the ACTA2 ${ }^{-/-}$mouse aorta

One $\mathrm{ACTA2}^{-/-}$mouse died on day 17 of the experiment, in response to AngII stimulation. On average, the aortas from $\mathrm{ACTA}^{2 /-}$ mice had significantly larger inner diameters than those from AngII stimulated WT mice $(\mathrm{n}=10, \mathrm{P}<0.05)$. As expected, the aorta of AngII treated mice were significantly more dilated than those of the WT mice $(\mathrm{n}=10, \mathrm{P}<0.01$; Figure 1).

\section{Increased OPN expression and decreased $\alpha$-smooth muscle actin ( $\alpha-S M A)$ expression in the AngII-treated ACTA2 $2^{--}$ mouse aortic wall}

The expression levels of OPN and $\alpha$-SMA in the mouse aortic wall tissue from WT, AngII stimulated WT (AngII) and $\mathrm{ACTA2}^{-/-}$mice (ACTA2) were evaluated by Western blot. As displayed in Figure 2, in ACTA2 ${ }^{-/}$aortic walls, the OPN expression was increased and $\alpha$-SMA was decreased compared to the levels of expression in the other two groups (Figure 2). The expression levels of $\alpha$-SMA were also evaluated using RT-PCR (Figure 3). There was a significant difference in the expression levels of $\alpha$-SMA between the AngII and the ACTA2 group $(0.86 \pm 0.07$ vs. $0.46 \pm 0.02$, $\mathrm{P}<0.01)$ as well as between the WT and the AngII groups $(1.39 \pm 0.11$ vs. $0.86 \pm 0.07, \mathrm{P}<0.05)$. These findings suggest that, in the ACTA2 knockout, there was a phenotypic modulation in VSMCs from the contractile phenotype to the secreting phenotype.

\section{ACTA2 deficiency rendered aortic SMC susceptible to apoptosis}

We first evaluated the expression of Bax and Bc-2 by Western blot. Both of these proteins mediate apoptosis (Figure 4A). Mice in the AngII group had increased Bax expression and decreased $\mathrm{Bcl}-2$ expression compared with the expression in the WT mice $(\mathrm{n}=3, \mathrm{P}<0.05)$. Conversely, the expression level of these two genes were similar between the WT group and the NS group $(\mathrm{n}=3, \mathrm{P}<0.001)$. Apoptosis was evaluated by TUNEL staining. As shown in Figure $4 B$ and $4 \mathrm{C}$, the aorta of ACTA2 mice had more apoptotic cells than the aorta of the AngII or WT mice $(\mathrm{n}=6, \mathrm{P}<0.05)$. As expected, the level of apoptotic cells in the aortas of AngII mice was higher than that of the WT mice $(n=6, P<0.01)$. These findings revealed that ACTA2 deficiency renders aortic SMC susceptible to apoptosis, at least partially through mediating the expression levels of Bax and Bcl-2.

\section{AngII stimulated ACTA2 ${ }^{-/-}$mice had increased collagen fiber level and decreased elastin level and integrity in the aortic walls}

We next examined aortic collagen deposition and elastin 


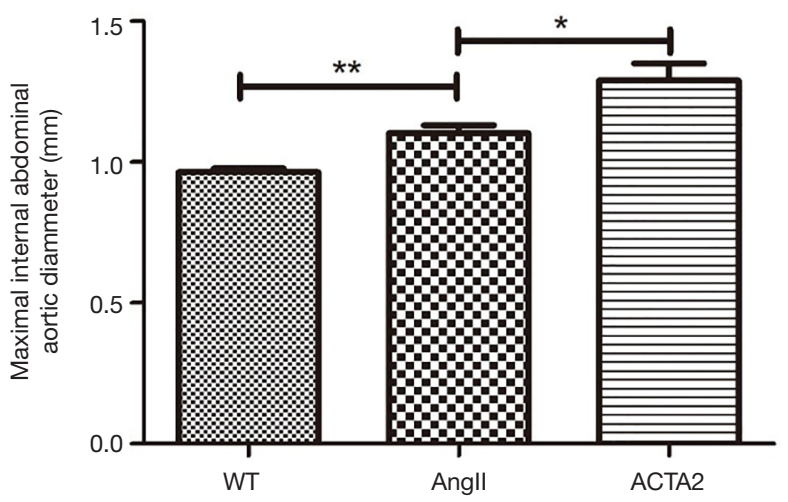

Figure $1 \mathrm{ACTA2}^{-/-}$mice heightened AngII induced progressive lumen dilation of the aortas. Data are expressed as mean \pm SEM. $\mathrm{N}=10$ per group. *, $\mathrm{P}<0.05 ;{ }^{* *}, \mathrm{P}<0.01$. AngII, angiotensin II; WT, wild-type; ACTA2, smooth muscle actin alpha 2; SEM, standard error of the mean.

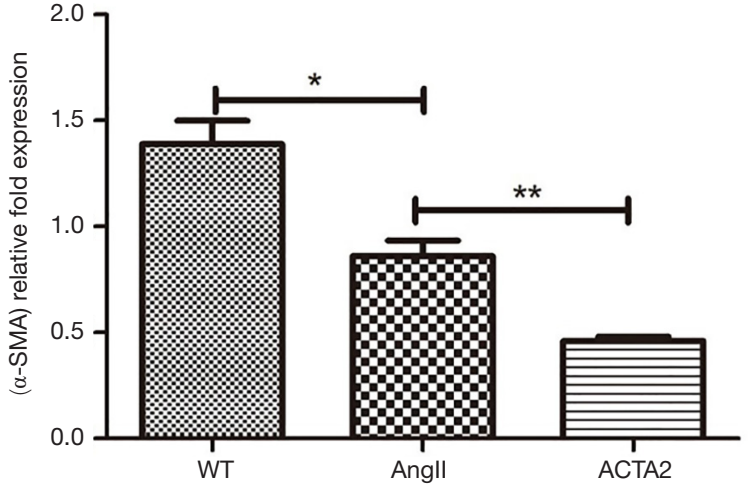

Figure $3 \alpha$-SMA mRNA was significantly decreased in AngII stimulated $\mathrm{ACTA}^{-/-}$mouse aorta. Real-time PCR was performed as described in materials and methods. Data are expressed as the mean \pm SEM. $\mathrm{n}=3$ per group. *, $\mathrm{P}<0.05$; **, $\mathrm{P}<0.01$. AngII, angiotensin II; WT, wild-type; ACTA2, smooth muscle actin alpha 2; $\alpha$-SMA, $\alpha$-smooth muscle actin; PCR, polymerase chain reaction; SEM, standard error of the mean.
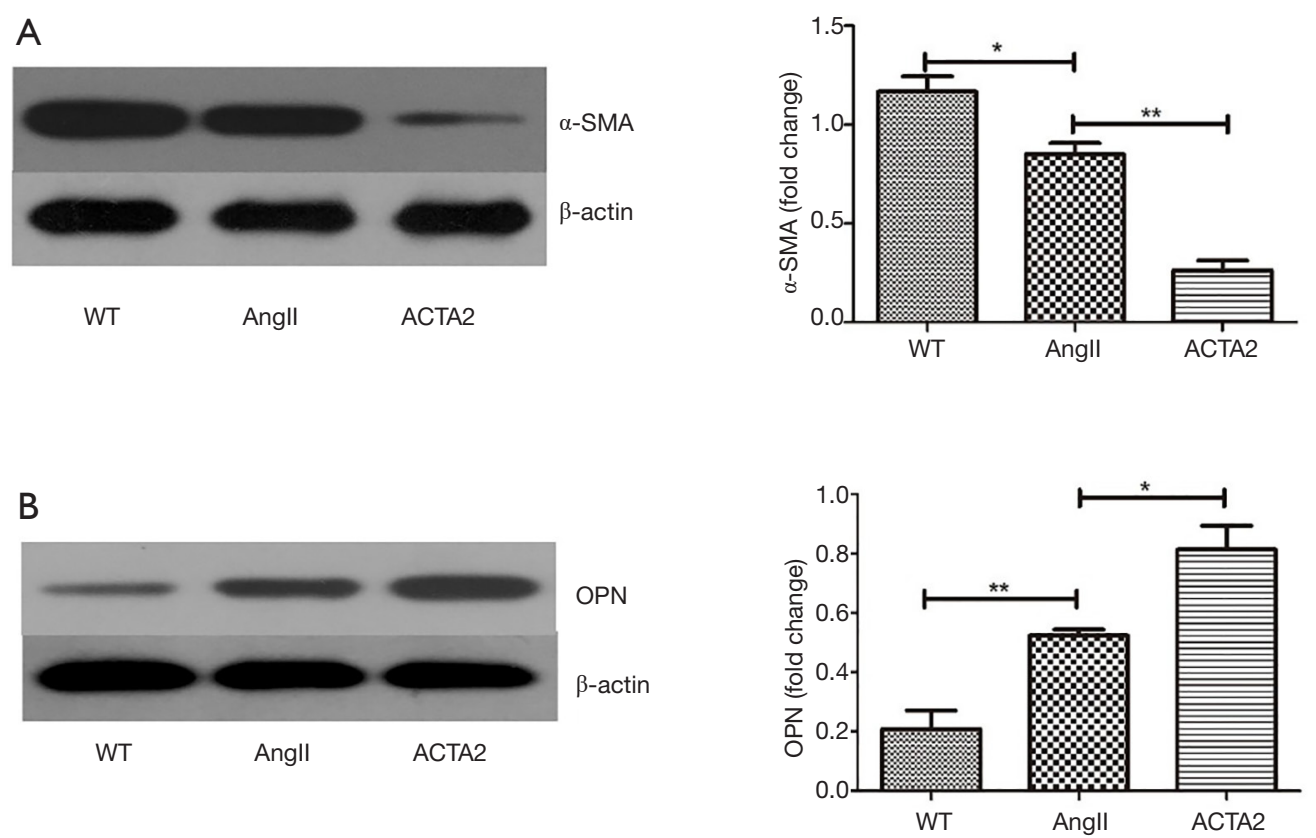

Figure 2 Increased OPN expression and decreased $\alpha$-SMA expression in AngII-treated ACTA2 ${ }^{-/}$mouse aortic wall. Western blot was performed for $\alpha$-SMA (A) and OPN (B) on protein lysates purified from aorta of mice from WT, AngII and ACTA2 ${ }^{-/-}$group. Data are expressed as the mean \pm SEM. $\mathrm{n}=3$ in each group. * $\mathrm{P}<0.05$; ** $\mathrm{P}<0.01$. OPN, osteopontin; $\alpha$-SMA, $\alpha$-smooth muscle actin; AngII, angiotensin II; WT, wild-type; ACTA2, smooth muscle actin alpha 2; SEM, standard error of the mean. 
A

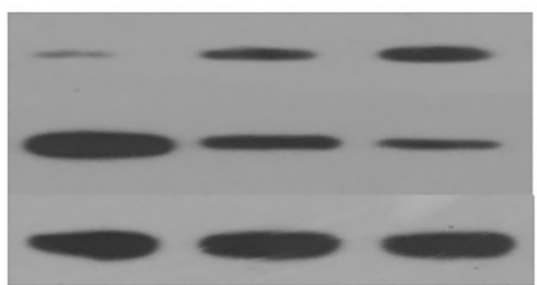

WT

Angll

ACTA2

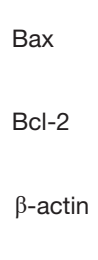

$\beta$-actin

$$
\text { Angl }
$$

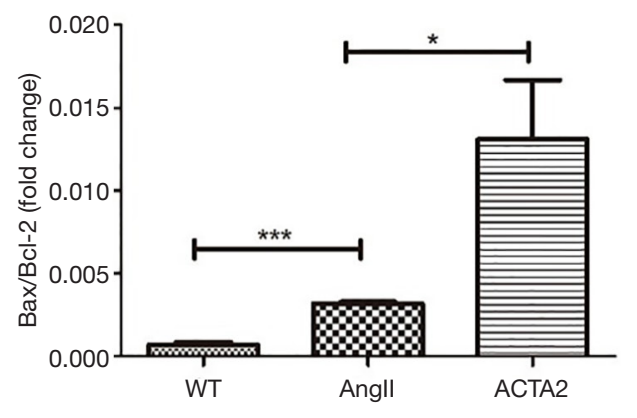

B

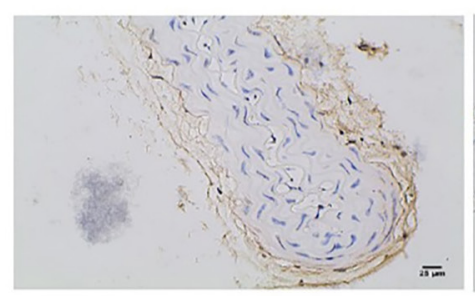

WT

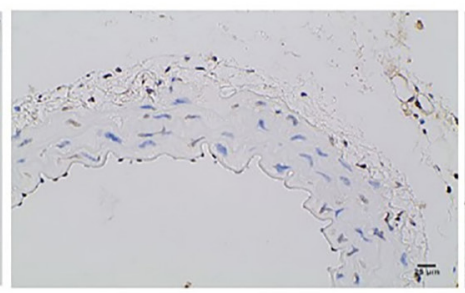

Angll

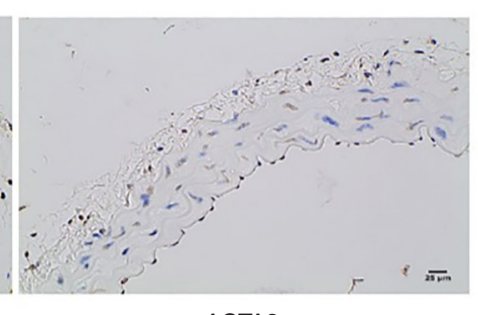

ACTA2

C

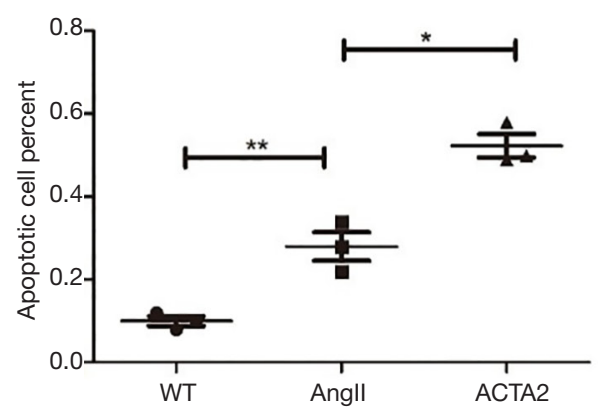

Figure 4 ACTA2 deficiency rendered aortic SMC susceptible to apoptosis. The expression levels of Bax and Bcl-2 were assessed by western blot (A); apoptosis was evaluated by TUNEL stain (B,C). Data are expressed as mean \pm SEM. N=3 per group. The meaning of Bax/Bcl2 increasing is that Apoptosis increases. *, $\mathrm{P}<0.05$; **, $\mathrm{P}<0.01$. SMC, smooth muscle cell; AngII, angiotensin II; WT, wild-type; ACTA2, smooth muscle actin alpha 2; SEM, standard error of the mean.

expression in the aortic walls of WT, AngII, and ACTA2 mice by $\mathrm{H} \& \mathrm{E}, \mathrm{VVG}$, and Masson's staining. As shown in Figure 5, in the AngII and ACTA2 mice more elastic laminae thickening and aortic ruptures were observed than in the WT mice, with the thickest laminae being observed in the ACTA2 mice. Additionally, the order of the collagen fiber levels in the aorta walls was as follows: ACTA2 > AngII $>$ WT.

\section{Discussion}

Infusion of mice with AngII has been a very valuable model to aid in researching the pathogenesis and formation of aortic aneurysms and dissections (7). Our study mainly used this model to explore the role of ACTA2 in the pathogenesis of thoracic aortic aneurysms and dissections.

The tunica media of an artery consists mainly of extracellular matrix (ECM) and VSMCs. Under physiological conditions, both ECM and VSMCs contribute to the elasticity and tensile strength of the tunica media to maintain integrity and contractility of the aortic wall (8). In the artery of aortic dissections, VSMCs exhibited increased apoptosis (9), which was evidenced by the accumulation of p53 in the cell nucleus of VSMCs and the higher 


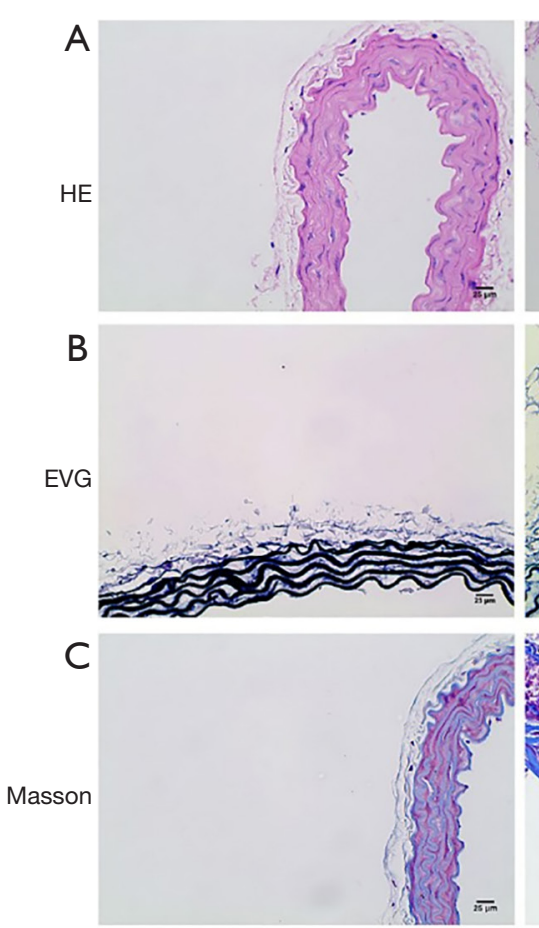

WT

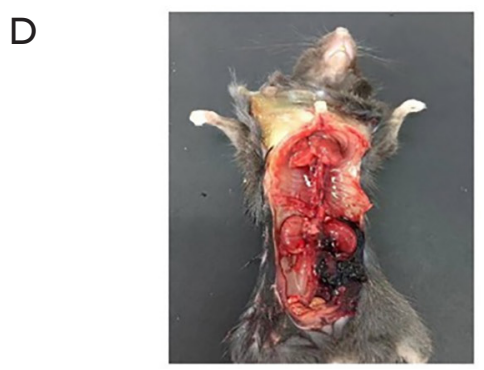

ACTA2
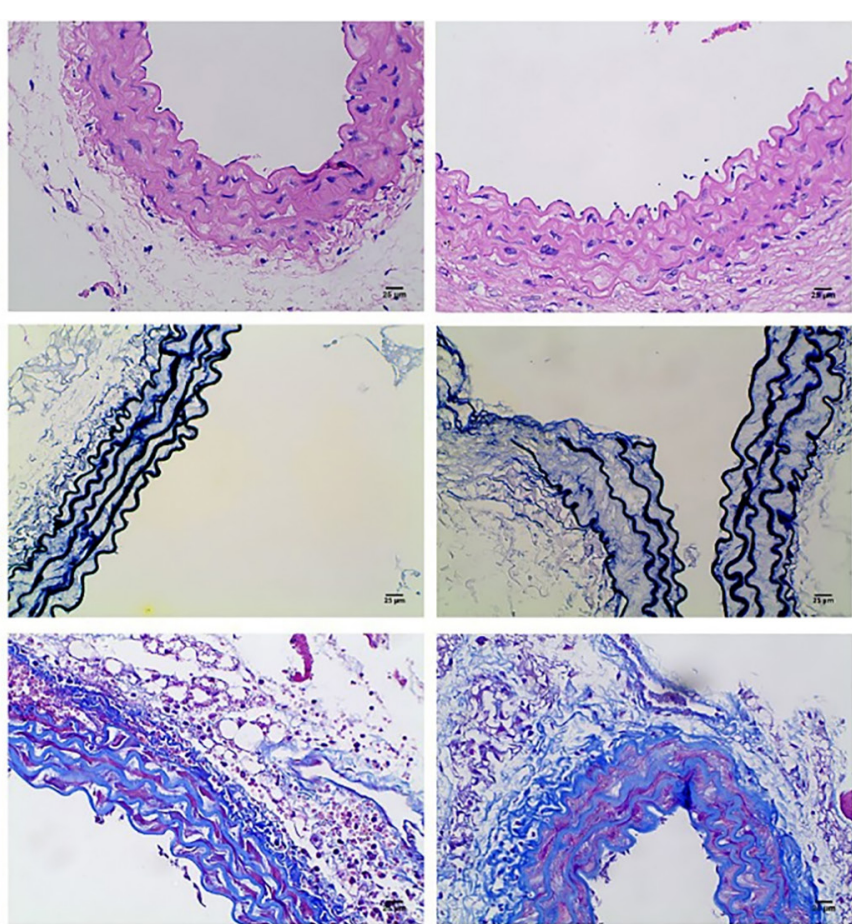

Angll

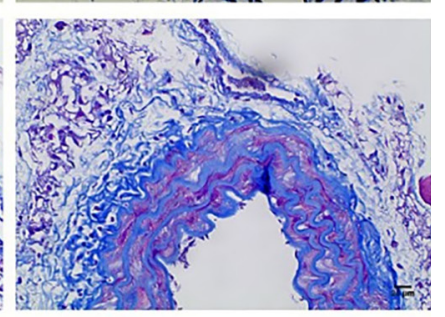

ACTA2

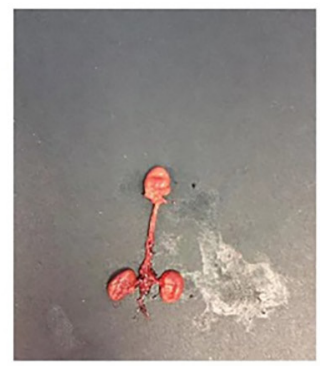

ACTA2

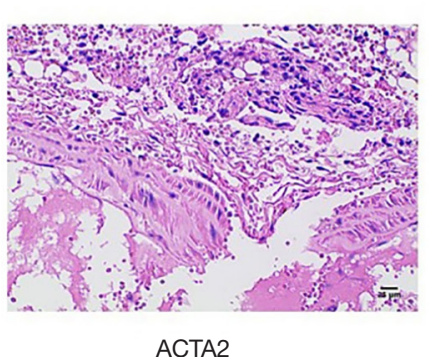

ACTA2

Figure 5 AngII stimulated ACTA2 ${ }^{-/-}$mice had increased collagen deposit and decreased elastin level and integrity in the aortic walls. (A) $\mathrm{H}$ \& E staining of aorta (bar, $25 \mu \mathrm{m}$ ); (B) VVG staining of aorta (bar, $25 \mu \mathrm{m}$ ); (C) Masson staining of aorta (bar, $25 \mu \mathrm{m})$; (D) images showing aortic dissection of ACTA2 ${ }^{-/-}$mice, H \& E staining of aorta (bar, $25 \mu \mathrm{m}$ ). VVG, Verhoeff-Van Gieson; H\&E, hematoxylin and eosin; AngII, angiotensin II; WT, wild-type; ACTA2, smooth muscle actin alpha 2; SEM, standard error of the mean.

expression of gene BAX, which contributes to apoptosis in the ECM (10). Increased VSMC apoptosis has been demonstrated to be related to the expansion of the tunica media of an artery, increased rupture of the elastic layer, and abnormal deposition of ECM (11). In the present study, we demonstrated that VSMC apoptosis in the aortic wall in response to AngII stimulation was increased in ACTA2 mice. These findings indicate that VSMC apoptosis is critical in the remodeling of the tunica media of an artery, which may result in aortic aneurysms and dissections.

The phenotypes of VSMCs include the contractile and secreting phenotypes. A previous study investigating Marfan syndrome using mouse models revealed phenotypic modulation of VSMCs, i.e., a change from the contractile type to the secreting type (12). Evidence of this transformation was found in the myofilament loss of VSMCs. In addition, the genes which encode cytoskeleton and myofibril proteins were found to be downregulated, thus promoting the formation of extensive rough endoplasmic reticuli and grand Golgi complexes (13-15). The decrease in the expression of cytoskeleton and myofibril proteins hinders cell migration. The new 
synthetic rough endoplasmic reticuli and grand Golgi complexes cause the formation of excessive remodeling substance of the aortic wall. Therefore, elucidation of the mechanism of VSMC apoptosis and transformation of their phenotype may be the key to identifying the pathogenesis of thoracic aortic aneurysms and dissections.

Alpha-SMA is a significant marker of the contractile phenotype of VSMCs (16), while OPN is a marker of the secreting phenotype of VSMCs (17). In the present study, we found that, in ACTA2 $2^{--}$mouse aorta, $\alpha$-SMA expression was decreased while OPN expression was increased, suggestive of a phenotypic transformation from the contractile to the secreting phenotype. Previous studies have demonstrated that treatment of VSMCs with OPN significantly increased the formation of autophagosomes, the expression of autophagy-related genes, and cell death. Furthermore, blocking the OPN signal, with an anti-OPN antibody, markedly inhibited OPN-induced autophagy and VSMC death (18). Taken together, the above observations of the suppression of OPN activation suggest that this may be a viable option for the treatment of thoracic aortic aneurysms and dissections.

Most of the VSMCs in the normal vascular wall of an aorta are contractile, which requires the elasticity of the vascular wall. However, VSMCs may transform from a contractile phenotype to a secreting phenotype after vascular injury or if the vascular wall is struck forcibly. Thereafter, VSMCs can secrete a large amount of ECM, resulting in increased cell proliferation and migration. Thus, the phenotypic modulation in VSMCs plays a significant role in the repair of vascular injury. These same cells may also release an important initial sign relating to pathogenic changes. In addition, proliferative VSMCs secrete a large amount of ECM, which eventually forms atherosclerosis $(19,20)$. Consistent with these reports, we observed increased collagen deposition in the AngII stimulated $\mathrm{ACTA}^{-/-}$mouse aorta, suggesting that phenotypic modulation in VSMCs of the aortic wall may be an important indicator for early atherosclerosis.

Previous studies have demonstrated increased apoptosis in VSMCs in cases of aortic aneurysms compared with the VSMCs of a normal aorta $(21,22)$. Additionally, an increased ratio of $\mathrm{Bax} / \mathrm{Bcl}-2$ promotes apoptosis, while a decrease of this ratio inhibits apoptosis (23). In agreement with these observations, we found significantly increased apoptosis in VSMCs that were stimulated with AngII compared with NS-treated VSMCs. Moreover, ACTA2 deletion further exacerbated the AngII-triggered apoptosis. This increase in apoptosis coincided with an increased ratio of $\mathrm{Bax} / \mathrm{Bcl}-2$. Thus, we believe that ACTA2 is involved in the mediation of AngII induced apoptosis, at least in part through an alteration in the expression of Bax and Bcl-2.

\section{Study limitations}

The role of ACTA2 in the formation of thoracic aortic aneurysms and dissections should be examined further in a gain-of-function mouse model.

\section{Conclusions}

We report here that knockout of ACTA2 contributes to the formation of thoracic aortic aneurysms and dissections in the presence of AngII. Knockout of ACTA2 also enhances phenotypic modulation and apoptosis mediated by AngII in VSMCs, which in turn makes the vascular wall more vulnerable and may lead to vascular rupture. Our findings suggest that the treatment of patients with ACTA2 gene therapy may aid in preventing the formation of thoracic aortic aneurysms and dissections. Thus, our observations provide rationale for the targeting of ACTA2 as a potential novel treatment of thoracic aortic aneurysms and dissections.

\section{Acknowledgements}

Funding: This study was funded by the National Nature Science Foundation of China (No.81370416).

\section{Footnote}

Conflicts of Interest: The authors have no conflicts of interest to declare.

Ethical Statement: All animal procedures were approved by the Committee Tongji Medical College, Huazhong University of Science and Technology (ethical card: IACUC No. 630).

\section{References}

1. Lilienfeld DE, Gunderson PD, Sprafka JM, et al. Epidemiology of aortic aneurysms: I. Mortality trends in the United States, 1951 to 1981. Arteriosclerosis 1987;7:637-43.

2. Milewicz DM, Michael K, Fisher N, et al. Fibrillin-1 
(FBN1) mutations in patients with thoracic aortic aneurysms. Circulation 1996;94:2708-11.

3. Guo DC, Pannu H, Tran-Fadulu V, et al. Mutations in smooth muscle alpha-actin (ACTA2) lead to thoracic aortic aneurysms and dissections. Nat Genet 2007;39:1488-93.

4. Morisaki H, Akutsu K, Ogino H, et al. Mutation of ACTA2 gene as an important cause of familial and nonfamilial nonsyndromatic thoracic aortic aneurysm and/ or dissection (TAAD). Hum Mutat 2009;30:1406-11.

5. Regalado ES, Guo DC, Prakash S, et al. Aortic Disease Presentation and Outcome Associated With ACTA2 Mutations. Circ Cardiovasc Genet 2015;8:457-64.

6. Bellini C, Wang S, Milewicz DM, et al. Myh11(R247C/ $\mathrm{R} 247 \mathrm{C})$ mutations increase thoracic aorta vulnerability to intramural damage despite a general biomechanical adaptivity. J Biomech 2015;48:113-21.

7. Kelleher CM, McLean SE, Mecham RP. Vascular extracellular matrix and aortic development. Curr Top Dev Biol 2004;62:153-88.

8. Humphrey JD. Possible mechanical roles of glycosaminoglycans in thoracic aortic dissection and associations with dysregulated transforming growth factorbeta. J Vasc Res 2013;50:1-10.

9. Watanabe M, Sawai T. Alteration of cross-linking amino acids of elastin in human aorta in association with dissecting aneurysm: analysis using high performance liquid chromatography. Tohoku J Exp Med 1999;187:291-303.

10. Karnik SK, Brooke BS, Bayes-Genis A, et al. A critical role for elastin signaling in vascular morphogenesis and disease. Development 2003;130:411-23.

11. Rodgers UR, Weiss AS. Integrin alpha v beta 3 binds a unique non-RGD site near the C-terminus of human tropoelastin. Biochimie 2004;86:173-8.

12. Mochizuki S, Brassart B, Hinek A. Signaling pathways transduced through the elastin receptor facilitate proliferation of arterial smooth muscle cells. J Biol Chem
2002;277:44854-63.

13. Wagenseil JE, Nerurkar NL, Knutsen RH, et al. Effects of elastin haploinsufficiency on the mechanical behavior of mouse arteries. Am J Physiol Heart Circ Physiol 2005;289:H1209-17.

14. Li DY, Faury G, Taylor DG, et al. Novel arterial pathology in mice and humans hemizygous for elastin. J Clin Invest 1998;102:1783-7.

15. Kielty CM, Baldock C, Lee D, et al. Fibrillin: from microfibril assembly to biomechanical function. Philos Trans R Soc Lond B Biol Sci 2002;357:207-17.

16. Regan CP, Adam PJ, Madsen CS, et al. Molecular mechanisms of decreased smooth muscle differentiation marker expression after vascular injury. J Clin Invest 2000;106:1139-47.

17. Feil S, Hofmann F, Feil R. SM22alpha modulates vascular smooth muscle cell phenotype during atherogenesis. Circ Res 2004;94:863-5.

18. Zheng YH, Tian C, Meng Y, et al. Osteopontin stimulates autophagy via integrin/CD44 and p38 MAPK signaling pathways in vascular smooth muscle cells. J Cell Physiol 2012;227:127-35.

19. Owens GK, Kumar MS, Wamhoff BR. Molecular regulation of vascular smooth muscle cell differentiation in development and disease. Physiol Rev 2004;84:767-801.

20. Majesky MW. Developmental basis of vascular smooth muscle diversity. Arterioscler Thromb Vasc Biol 2007;27:1248-58.

21. Lopez-Candales A, Holmes DR, Liao S, et al. Decreased vascular smooth muscle cell density in medial degeneration of human abdominal aortic aneurysms. Am J Pathol 1997;150:993-1007.

22. Thompson RW, Liao S, Curci JA. Vascular smooth muscle cell apoptosis in abdominal aortic aneurysms. Coron Artery Dis 1997;8:623-31.

23. Shtilbans V, Wu M, Burstein DE. Evaluation of apoptosis in cytologic specimens. Diagn Cytopathol 2010;38:685-97.
Cite this article as: Cheng J, Zhou X, Jiang X, Sun T. Deletion of ACTA2 in mice promotes angiotensin II induced pathogenesis of thoracic aortic aneurysms and dissections. J Thorac Dis 2018;10(8):4733-4740. doi: 10.21037/jtd.2018.07.75 